\title{
Les actualités de l'Institut UPSA de la douleur
}

\author{
The Latest News from the UPSA Pain Institute
}

\section{Nouvelle brochure pour les patients : « J'ai mal à la main, j'en parle »}

Rhumatisme, maladie de Raynaud, syndrome du canal carpien, tendinite, maladie de Dupuytren, kyste synovial... Tant de causes possibles de douleurs au niveau des mains.

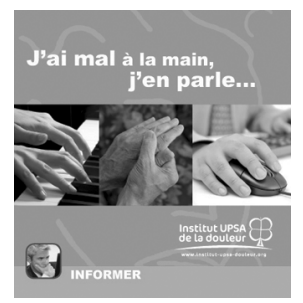

Cette brochure contribuera à répondre aux questions que peuvent se poser les patients ayant des douleurs des mains, les aidera à mieux comprendre leur douleur et les encouragera à consulter leur médecin ou à en parler à leur pharmacien.

\section{Quelques exemples}

Quelle douleur peut-on ressentir au niveau des mains? Quels maux peuvent survenir ? Quels en sont les symptômes ? Comment prévenir ces maux ? Comment les soigner ? À qui en parler?

Cette brochure sur les douleurs de la main vient compléter la collection de fascicules de l'Institut UPSA de la douleur conçus pour les patients et le grand public.

Pour mettre à disposition cette brochure dans les salles d'attente ou la distribuer, les professionnels de santé peuvent la commander, à titre gracieux, via le site Internet de l'Institut UPSA de la douleur : www.institut-upsa-douleur. org ou en adressant un e-mail à institut.upsa@bms.com.

\section{Nouveau numéro de la publication « La douleur, des recommandations à la pratique " : les douleurs de croissance, mythe ou réalité ?}

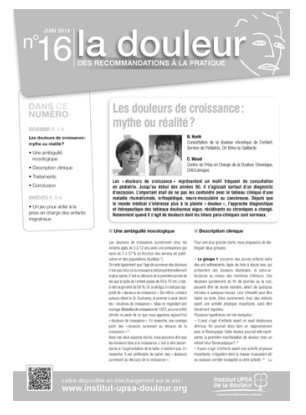

Qu'est-ce que les douleurs de croissance ? Qui est touché ? Comment décrire ces douleurs? Quels sont les traitements?

Les douleurs de croissance sont un motif fréquent de consultation en pédiatrie. Ces douleurs surviennent chez les enfants de 3 à 12 ans. L'âge où surviennent les douleurs n'est pas celui où la croissance est proportionnellement la plus rapide. C'est au décours de la première année de vie que la taille de l'enfant passe de 50 à $75 \mathrm{~cm}$, donc augmente de $50 \%$.

\section{Douleurs différentes en fonction de l'âge de l'enfant}

- Les jeunes enfants, voire des préadolescents, qui présentent des douleurs bilatérales et extra-articulaires au niveau des membres inférieurs. Ces douleurs surviennent en fin de journée ou la nuit. Leur intensité peut être faible ou forte. Elles surviennent chez des enfants ayant une activité physique importante.

- Les enfants âgés de 8 à 14/15 ans ayant une activité sportive extrascolaire régulière qui doit être adaptée à la morphologie et à la capacité musculaire de l'enfant en sachant qu'il s'agit d'un organisme en cours de croissance.

\section{Traitements}

La prise en charge thérapeutique repose sur une association de traitements médicamenteux et non médicamenteux. Avant toute chose, il faut informer l'enfant et ses parents du diagnostic et expliquer « pourquoi cela fait mal, voire très mal ». L'impact thérapeutique de l'information et de la « démystification du problème » n'est pas négligeable, c'est d'ailleurs un des pivots de la compréhension du problème et de l'éducation thérapeutique.

\section{Conclusion}

Les douleurs musculaires et ostéotendineuses sont un motif fréquent de consultation en médecine générale, en pédiatrie et en orthopédie pédiatrique. Chez le petit enfant ou le préadolescent qui n'a pas une activité physique régulière, les douleurs sont plutôt d'origine musculaire. Les enfants et adolescents qui ont une activité sportive régulière, voire très dense, sont davantage exposés à des douleurs ostéoarticulaires ou tendineuses. En cas de douleur articulaire chez un enfant sportif, on conseillera donc plutôt un arrêt temporaire des activités sportives.

\section{Où trouver ces documents ?}

Cette brochure et cette publication, comme l'ensemble de la documentation proposée par l'institut, sont disponibles gracieusement en libre téléchargement sur le site Internet de l'Institut UPSA de la douleur : www.institut-upsa-douleur.org. 